\title{
Pancreas Versus Islets After a Successful Kidney Transplant
}

\author{
Boris L. Gala-Lopez • Andrew R. Pepper • \\ A. M. James Shapiro
}

Published online: 1 April 2014

(C) Springer International Publishing AG 2014

\begin{abstract}
The optimal endocrine transplant that should follow a successful kidney transplant in patients with type 1 diabetes is currently open to debate. Ideally, a combined kidney and pancreas transplant is carried out simultaneously from an optimal donor as there is clear immunological benefit. However, when a living donor kidney transplant occurs initially, either a pancreas after kidney or islet after kidney transplant may be considered. A pancreas after kidney transplant carries more surgical risk but provides more robust endocrine reserve compared to the alternative option of islet after kidney transplantation. Furthermore, islet transplantation is not universally available, requires specialized manufacturing facilities, and the costs associated with islet manufacture may not be reimbursable by healthcare. From a patient's perspective, however, islet after kidney transplantation is a highly attractive option to avoid further surgical risk and recovery. This paper discusses the merits and demerits of these alternative transplant options, and debates both competitive aspects and complementarity. We discuss current outcomes and ongoing clinical trials.
\end{abstract}

Keywords Diabetes mellitus · Pancreas transplantation - Islet transplantation $\cdot$ Renal insufficiency $\cdot$ Kidney transplantation

\begin{tabular}{ll}
\multicolumn{2}{l}{ Abbreviations } \\
T1DM & Type 1 Diabetes Mellitus \\
SPK & Simultaneous Pancreas-Kidney \\
SIK & Simultaneous Islet-Kidney \\
PAK & Pancreas After Kidney \\
IPTR & International Pancreas Transplant Registry
\end{tabular}

B. L. Gala-Lopez · A. R. Pepper · A. M. J. Shapiro $(\varangle)$

Clinical Islet Transplant Program and Department of Surgery,

University of Alberta, 2000 College Plaza, 8215 112th St, Edmonton,

AB T6G 2C8, Canada

e-mail:amjs@islet.ca

$\begin{array}{ll}\text { BMI } & \text { Body Mass Index } \\ \text { DRI } & \text { Donor Risk Index } \\ \text { SRTR } & \text { Scientific Registry of Transplant Recipients } \\ \text { MMF } & \text { Mycophenolate Mofetil } \\ \text { mTOR } & \text { Mammalian Target of Rapamycin Inhibitors } \\ \text { CITR } & \text { Collaborative Islet Transplant Registry } \\ \text { ITA } & \text { Islet Transplant Alone } \\ \text { OPTN } & \text { Organ Procurement and Transplantation Network } \\ \text { QALY } & \text { Quality-Adjusted Life Years } \\ \text { FDA } & \text { Food and Drug Administration } \\ \text { IND } & \text { Investigational New Device }\end{array}$

\section{Introduction}

The optimal management of patients with type 1 diabetes (T1DM) that have previously received a successful kidney transplant is hotly debated. A poor option is to offer no further transplant, as without correction of the underlying $\beta$-cell deficiency, kidney transplant graft half-life is shortened substantially, and overall patient survival is curtailed $[1,2]$. Accelerated diabetic nephropathy compounded by calcineurin inhibitor toxicity leads to worse long term outcomes in the absence of endocrine $\beta$-cell transplantation, and the inexorable systemic attrition from advancing macro- and microangiopathic secondary diabetic complications further shorten lifespan. Two alternative $\beta$-cell transplant options exist currently, including pancreas after kidney transplantation (PAK) or islet after kidney transplantation (IAK). Which should be offered when and to whom, is open to much discussion.

Ideally, a combined kidney and pancreas transplant is carried out simultaneously from an optimal donor as there is clear immunological benefit. Correction of hyperglycemia with restoration of physiologic, regulated internal insulin and counter regulatory hormonal release lowers post-operative 
complications and improves both short and long term graft survival $[3-5,6 \bullet]$.

Simultaneous islet-kidney transplantation (SIK) may also be considered, but is often not feasible because of the challenges involved in islet isolation, which may fail to yield sufficient islets for transplantation $[6 \cdot, 7,8]$. It is clear from renal transplant data that both short-term and long-term transplant outcomes are optimal when a living donor kidney transplant is selected over deceased donor kidney transplantation [9•]. Selected centers, notably the University of Maryland, have promoted simultaneous cadaveric pancreas with living donor (laparoscopic) kidney transplantation (SPLK), a technique that provides optimal graft function and utilization, while minimizing inductive rounds of immunosuppression $[10,3]$. There are clearly logistical challenges both for the center and the potential living donor with this approach, but the results are most encouraging with 1-year pancreas survival superior to SPK ( $88 \%$ vs. $84 \%)$ and similar morbidity and rejection rates (23\% vs. $24 \%)[3,10]$. Such an approach would also be an attractive consideration for simultaneous islet and living donor kidney (SILK), and would carry increased practicality compared with SPLK, as the donor laparoscopic nephrectomy could be done in a more relaxed day-time 'elective' setting, while the islets are maintained in culture, and again, just a single round of inductive immunosuppression would be required.

When the kidney transplant is performed first, either a PAK or IAK transplant may be considered. A PAK transplant carries more surgical risk but generally provides higher endocrine reserve compared to the alternative option of IAK transplantation. Furthermore, and perhaps most importantly from a treatment delivery perspective, islet transplantation is not universally available, requires specialized manufacturing facilities, and costs associated with islet manufacture can be prohibitive. In the United States (US), islet transplantation is currently regarded as 'experimental' by the Food and Drug Administration (FDA), and all allogenic islet transplants must be carried out under an Investigational New Device (IND) application. Until a biological license is granted, islet transplantation will not be reimbursable by third party payers in the US. This is in distinct contrast to the situation in Canada, the United Kingdom, Australia, Sweden, Switzerland, France, Italy and other European countries where islet transplantation is now governmentfunded and provided as part of standard 'non-research' care. From a patient's perspective, however, islet after kidney transplantation remains a highly attractive approach for avoidance of further surgical risk and recovery. This paper discusses the merits and demerits of these diametrically opposed transplant options, and highlights both competitive and complementary aspects. We discuss current outcomes and ongoing clinical trials.

\section{Recent Outcomes Have Improved Substantially in Both Pancreas and Islet Transplantation}

Considerable advances have occurred in both the technical application and clinical outcomes of whole pancreas transplantation since its introduction by Kelly and Lillehei in 1966. Enteric exocrine drainage has reduced high rates of complications from bladder drainage. More potent T-depletional induction agents combined with calcineurin inhibitor-based maintenance immunosuppression have reduced or eliminated the need for high-dose corticosteroids $[11 \bullet \bullet, 12,13]$. This has resulted in marked reduction in rates of acute rejection, while at the same time dramatically reduced risk of surgical-site sepsis and life-threatening opportunistic infections. Reduced risk of acute rejection has allowed safe transition from bladder to enteric drainage, as former reliance upon urinary amylase excretion as a marker of rejection is no longer required, being supplanted by serum amylase and lipase measurement. T cell depletional induction therapies with anti-thymocyte globulin, alemtuzumab or alternates have further markedly lowered rejection rates and improved long term insulin-independent clinical outcomes, possibly through improved control of islet autoimmunity [14-16]. Portal venous drainage is used by selected centers and may improve physiological metabolic function on stimulation testing, but overall glycemic control is similar. Portal venous drainage has been associated with lower rejection rates in some reports, but is not an essential requirement, and may be associated with slightly increased technical complication rates [17, 18]. Pancreas transplantation has been carried out worldwide in more than 35,000 cases over the past 35 years, and has proven success in restoring normoglycemia with insulin independence and sustained normal hemoglobin A1C. Given the inherited surgical risk associated with whole pancreas transplantation, the procedure is largely carried out in patients with T1DM and associated endstage renal failure, where a simultaneous transplant can provide both freedom from dialysis and insulin, while minimizing risk of new-onset diabetic vasculopathy affecting the new kidney [19].

Pancreas after kidney (PAK) transplant is being used increasingly in the current era of deceased donor shortage, where living donation has become a valuable additional resource for renal transplantation. Deleterious outcomes were previously attributable to PAK and pancreas transplant alone (PTA), where increased technical complication rates of vascular thrombosis and rejection were observed, associated with decreased pancreas graft survival [20•, 21, 22]. While immunological graft loss has been consistently higher both in PAK and PTA transplantation, this gap has closed considerably in the most recent era. Data provided by the International Pancreas Transplant Registry (IPTR) now demonstrates substantial improvement in 1-year graft survival of $85 \%$ vs. $80 \%$ for SPK and PAK (Fig. 1) [23••]. This improvement may be 


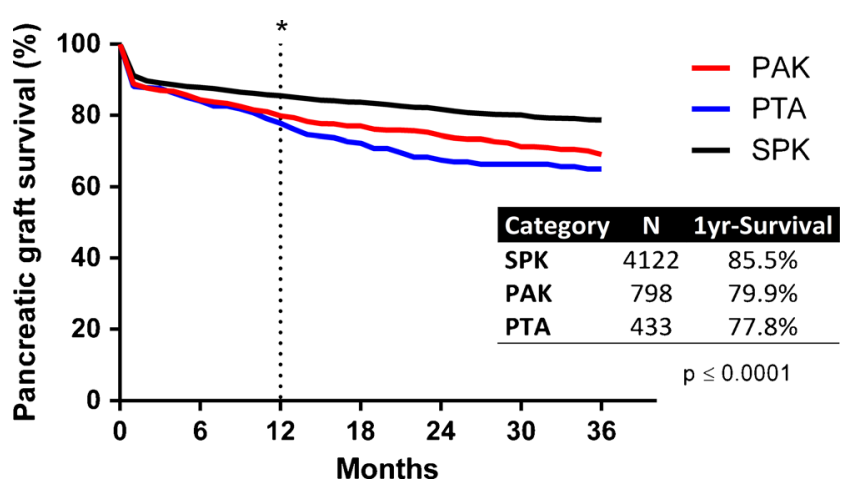

Fig. 1 Graft survival among adult pancreas transplant recipients in the US from $2005-2010$. All transplants are from deceased donors registered by IPTR/UNOS [23••]. This data is kindly reproduced with express permission from the IPTR registry

attributed largely to improved technical success coupled with more effective inductive and maintenance immunosuppression. The obvious advantages of a successful living donor kidney followed sequentially by PAK or IAK transplantation are that the recipient accrues additional benefit from earlier transplantation (less time on dialysis), and additional kidneys are thus available for other patients without recourse to living donors on the renal transplant waiting list. Non-sensitized patients have the flexibility to be listed as SPK and PAK, allowing dynamic allocation when a donor becomes available, and they may also have access to living donation. This approach further increases utilization of pancreas-alone grafts (including those sourced from donation after cardiac death) as and when suitable pancreata become available $[24,25,15]$.

Similarly, substantial advances have occurred in clinical islet transplantation over the past 14 years. The 'Edmonton Protocol' series of islet-alone transplants in patients with brittle T1DM led to high rates of insulin independence at 1 year, where two or more intraportal islet infusions were given, under the cover of IL-2 receptor blockade and low dose tacrolimus/sirolimus maintenance immunosuppression [26]. While rates of insulin independence decreased markedly by 3 - 5 years, almost three-quarters of patients continued to make sufficient endogenous regulated insulin to completely prevent hypoglycemic reactions and to correct $\mathrm{HbA} 1 \mathrm{C}$ below $6.5 \%[27,28]$. An international multicenter trial demonstrated reproducibility of the Edmonton Protocol, but also emphasized variability relating to site experience in both islet manufacture and clinical dosing of sirolimus [29]. More recently, over 1,085 islet transplants have been carried out, as documented by the Collaborative Islet Transplant Registry (CITR) [30••]. The latest CITR results have improved substantially, with 3-year rates of insulin independence improved from $27 \%$ to $44 \%$ in the most recent era [30••]. Bellin et al found that T-depletional induction and anti-inflammatory treatment with TNF $\alpha$ blockade with etanercept in the peritransplant period led to $50 \%$ insulin independence rates at 5 years, which match the results of pancreas-alone transplantation [31•]. Shapiro summarized results in at least six international islet transplant centers reporting similar high insulin independence rates at 5 years, suggesting parity of outcome of islet and pancreas-alone transplantation at 5 years in highly selected leading centers [32]. While the results of IAK transplantation appear to parallel the results of islet-alone transplantation at 3 years [33-35], longer term outcome data is clearly needed as the outcomes of PAK are still superior to PTA, and further robust studies are awaited to compare long term outcomes of IAK with PAK.

\section{Key Aspects for Success in Pancreas After Kidney Transplantation}

Various elements alter outcomes of PAK, including: donor risk, recipient selection, timing of transplant and immunosuppressive inductive and maintenance protocols.

\section{Donor Risk}

Donor selection and meticulous care in the surgical aspects of pancreas retrieval are critical for the success of PAK. A shift in deceased donor demographics from young motor vehicle accident victims with isolated head trauma to older donors succumbing to cerebrovascular accidents generally means that fewer ideal donors are available for whole pancreas transplantation. Donor age, cause of death, donor weight/body mass index (BMI), glycemic control, hemodynamic stability, and cold preservation time, all contribute to the overall donor risk, which may be scored objectively as the donor risk index (DRI) $[36,37]$. The DRI has been applied mainly in liver and kidney transplantation and correlates strongly with clinical outcome $[36,37]$. More recently, Axelrod and colleagues performed a detailed Scientific Registry of Transplant Recipients (SRTR)based multivariate analysis and confirmed the validity of pancreatic DRI for different transplant modalities, emphasizing the benefits of this tool in the more rational allocation of pancreata in whole pancreas transplantation [38•].

\section{Patient Selection}

The success of PT is critically dependent upon careful selection of recipients. Formal indications and contraindications for pancreas transplantation have been extensively discussed elsewhere, and reflect commonality of selection of ant recipient with complex multisystem dysfunction and diffuse macroand microvascular complications [39]. A controversial aspect of patient selection is recipient age, which may correlate with cumulative co-morbidities. A trend towards increased 
recipient age is now occurring in PT, and the IPTR data reflects an increase in age greater than 40 years. Improved cardiovascular screening with predictive short and longer term survival has helped considerably in this process. Despite this trend, recipient age remains a significant factor for increased risk of death after SPK, but a similar effect may not apply in PAK [20 ]. More transplant centers are now expanding recipient age as a strategy to cope with an aging population facing organ dysfunction. A recent report has emphasized expansion in recipient age for pancreas transplantation both in North America and Europe to $>50$ years, with comparable results to younger age groups where adequate patient selection is applied [40].

\section{Timing for Pancreas Transplant}

An unresolved aspect of PAK is optimal timing for the pancreas transplant after a successful kidney transplant. Ideally, PAK should be carried out early after successful kidney transplantation, possibly under the same inductive immunosuppressive cover. Avoidance of repeated rounds of T-depletion, elevated calcineurin inhibitor exposure or corticosteroid recycling could clearly reduce risks of opportunistic infection and malignancy in this population. When patients undergo kidney transplant from either a living or deceased donor, multiple factors often delay future planning of the pancreas transplant, including acute or chronic renal transplant rejection, calcineurin-inhibitor related nephrotoxicity, recurrent renal disease, donor vascular disease, active or opportunistic infection, cardiovascular events (acute myocardial infarction, congestive heart failure, stroke, or peripheral vascular disease), and malignancy [21]. Inexorable progression of diabetes is not uncommon and further endorgan complications may truncate the ability to proceed with PAK. Patients experiencing marked decrease in cardiac reserve or showing clear evidence of aortoiliac vascular disease will likely be denied further consideration of PAK [41].

\section{Immunosuppressive Approaches}

SPK transplantation is a highly immunogenic event, despite being immunocompromised by end-stage renal disease. While immunosuppressive approaches vary between centers and between countries, most use a combination of three or four drugs to achieve effective immune protection both in the inductive and early maintenance phase $[42 \bullet \bullet, 43,39]$. The introduction of calcineurin inhibitors (CNI), and especially tacrolimus, substantially increased long-term graft survival [44-46]. Recurrence of diabetic autoimmunity has been a poorly studied aspect in pancreas transplantation, but there is no doubt that this entity occurs, and may contribute to pancreas graft loss over time [47-50].

SPK transplantation has traditionally shown superior longterm outcomes than PAK or PTA, mainly due to more effective immune protection SPK transplants from surrogate monitoring of creatinine where simultaneous renal and pancreas rejection may occur [20 $[$.

The introduction of potent agents including tacrolimus, mycophenolate mofetil (MMF) and mammalian target of rapamycin (mTOR) inhibitors have all had considerable impact in improving pancreas graft outcome while reducing offtarget adverse events. Despite this, the risk of chronic allograft dysfunction remains a constant threat. As a result, biologic Tdepletional agents are often given in pancreas transplantation [51]. Formerly, agents such as OKT3 were used, which were associated with higher rates of CMV infection and posttransplant lymphoproliferative disorder. Less toxic replacements including anti-thymocyte globulin and alemtuzumab have been less toxic and less prone to off-target opportunistic infection. Other recent promising biologic agents include antiCD3 antibodies, anti-CD25 antibodies and, more recently, alemtuzumab and belatacept [51].

Presently, most pancreas transplant programs rely on potent induction agents usually with anti-thymocyte globulin or thymoglobulin, followed by tacrolimus and mycophenolate mofetil, and where corticosteroids are used, they may be

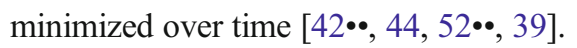

\section{Islet Transplantation in the Setting of Renal Failure}

The main indication for islet transplantation has been ITA in patients with T1DM associated with frequent hypoglycemia and severe glycemic lability. These indications mirror the indications for PTA. It took many years and considerable experimental effort to achieve efficient digestion and purification of human islets. The Ricordi Chamber together with a new class of highly purified enzyme blend has provided more reliable pancreatic dissociation, with enhanced islet yield, viability and function after islet purification on the COBE 2991 refrigerated centrifuge with continuous Biochrombased gradients [53, 54]. The Edmonton Protocol became a milestone in 2000 with the report of successful 1-year results in selected patients using ABO compatible, non-human leukocyte antigen matched islet grafts. The immunosuppressive protocol was modified to completely avoid corticosteroids, and exposure to calcineurin inhibitors was minimized through combination of low dose tacrolimus with sirolimus, together with daclizumab induction [54]. An essential component of this successful approach was the high-quality preparation of human islets from two or more donors in order to provide sufficient islet engraftment mass to cross the threshold requirement for insulin independence. 
Sustained C-peptide production and high early rates of insulin-independence after islet transplantation in T1DM has driven rapid proliferation of this technique around the world. Current results in islet transplantation are now possible after implementing newer, more potent immunosuppressant agents, avoidance of corticosteroids, and high-quality islet preparations; although, usually two islet infusions are required to attain insulin independence $[27,28,54,55 \cdot, 26,29]$.

The Edmonton group further reported that insulinindependence was not durable beyond 3 years in many patients, and most of the initial subjects needed to return to modest amounts of insulin over time, in the absence of recurrent hypoglycemia events [27, 28, 54, 55•, 26, 29]. A critical review of these data by the FDA suggested that protection from hypoglycemia and correction of HbA1C should be used as the primary therapeutic target rather than insulin independence, in trials addressing the impact of islet transplantation. Hence, in the large multicenter registration trials (CIT-07 and CIT-06) now progressing towards biological licensure, these positive outcomes have been selected by the FDA, and not insulin independence, as the major benefit of ITA.

Single-donor islet engraftment still remains a significant challenge for the islet field, and is one area that differs between islet and pancreas transplantation. This would maximize scare donor resource utilization, reduce potential risk of HLA sensitization [56, 32], and lower risk of donortransmitted disease (which has fortunately been exceedingly rare in islet transplantation to date [57, 58]). Ensuring high rates of single-donor engraftment in islet transplantation would require selective donor and recipient matching for size and insulin requirements, and a multimodal approach that protects islet mass throughout all stages of the procurement, isolation, culture, transplant and engraftment process. Strategies directed to suppress innate immunity and control inflammatory, immunological and thrombosis pathways in recipients are indeed showing promise, with increased 5-year graft durability and improved single-donor engraftment rates where such strategies have been engaged $[32,59]$.

Progress in small and large animal models has occurred in facilitating immunological tolerance with fewer complications. Nonetheless, such approaches have been hard to translate across to adult humans with a diverse immunological repertoire and memory. Novel approaches can facilitate islet engraftment and neovascularization, as well as new transplant sites to overcome current limitations of the standard intraportal engraftment site and allow the use of other alternatives like insulin-producing stem cells [60].

Despite the substantial transplant activity of many islet centers around the world, very few centers in North America are currently active in carrying out islet transplants. This largely reflects the experimental nature of islet transplantation in the US, and the acute shortage of allocated funds to conduct and support large scale trials. The Clinical Islet Transplant Program at the University of Alberta remains one of the most active islet transplant centers within the Collaborative Islet Transplant Registry (CITR), and in 2013, Edmonton carried out 66 islet transplants at a single center. Of over 200 patients transplanted with more than 400 intraportal islet preparations, the Edmonton group reports that currently $79 \%$ of patients continue to show full or partial islet graft function [61•]. The median duration of insulin independence is 34.6 and 11 months for subjects with full or partial graft function, whereas the duration of C-peptide is 53.3 and 70.4 months for those same patients $[5,62,63]$.

ITA has been particularly challenging in patients with preexisting marked diabetic renal dysfunction, as the ability of this group to tolerate high calcineurin inhibitor dosing may be severely compromised. Progressive renal dysfunction may occur from exposure to tacrolimus or sirolimus, which may compound ongoing renal damage from diabetic vasculopathy [64, 65]. Previously, the risk of broad HLA-sensitization has raised concerns about matching for future kidney transplantation, and the possibility of destabilization of a pre-existing renal allograft, possibly through HLA-priming or epitope spreading. The need for multiple islet donors may further compound this risk $[66,32]$. Fortunately the risk of HLA sensitization has only been of clinical significance in a small sub-group of islet recipients that have completely lost all islet graft function. Indeed, with T-depletional inductive immunosuppression and tacrolimus-based immunosuppression, this concern has largely been eliminated in recent islet transplant experience [32]. The risk has also been low in SIK transplantation $[56,67]$.

Recent reports comparing SPK and SIK have discussed potential advantages and disadvantages of islets versus pancreas transplantation. Gerber et al compared a 5-year cohort undergoing SPK or SIK in terms of long-term graft function and glucose control [6•]. Kidney function was similar between groups, but a higher rate of insulin-independence ( $96 \%$ vs. $31 \%$ ) was clearly observed in the SPK group. Despite this difference, SIK graft function was sufficient to maintain nearnormal glucose levels, and importantly was associated with significantly lower morbidity ( $0 \%$ vs. $40 \%$ relaparotomy rate in SPK). These findings led the authors to conclude that the endogenous insulin production resulting from SIK combined with optimal insulin therapy was sufficient to achieve satisfactory goals of glucose control, with avoidance of severe hypoglycemia with minimal complications. Similar reasoning may apply in IAK transplantation where patients have already undergone a successful kidney transplant, are obligatorily immunosuppressed, and then receive a minimally invasive cellular transplant procedure to assist with glycemic control. The clear benefit of IAK over PAK is the minimal risk associated with islet transplantation, and the possibility to consider patients for islet transplantation that would otherwise be 
discarded from PAK because of age or associated cardiovascular morbidity.

The risks associated with islet transplantation have also decreased markedly with cumulative center experience. Risks of bleeding following percutaneous intraportal islet transplantation may be minimized by effective ablation of the transhepatic catheter tract using thrombostatic paste of D-STAT or Avitene [68-71]. Risks of portal branch vein thrombosis have also been minimized through the combination of judicious portal pressure monitoring, use of highly purified islet preparations of low endotoxin content, combined with peritransplant therapeutic heparinization of the portal vein and the recipient [69]. Surgical access to the mesenteric portal tributaries may avoid these small risks, but requires a general anesthetic and is less attractive from a scar and recovery perspective.

From 1999 to 2009 the CITR reported 90/571 (15.7\% of the total islet procedures) with SIK or IAK (Table 1) [72]. Low numbers of IAK transplants compared with ITA make it hard to match outcomes with PAK currently. Deng et al reported comparable or superior islet graft in IAK versus ITA, even in the presence of low-dose corticosteroids [73]. A major trial of IAK is currently moving forward as part of the Collaborative Islet Transplant Consortium, designated CIT-06, and this trial will further add validation to biological licensure as the FDA reviews outcomes in these Phase 3 trials.

\section{Availability and Cost}

With over 35,000 cases worldwide, whole pancreas transplantation is an established therapy with over 1,000 procedures now being carried out per year [23••, 74]. Pancreas transplantation requires access to a fully trained pancreas transplant procurement and recipient transplant team, but the surgical aspects do not require specialized equipment that is not readily available at all transplant centers. Of the 247 active transplant centers in the United States, 143 have an active pancreas transplant program. In contrast, Canada has eight active pancreas transplant programs, and most transplants are carried out as SPK $[75,76]$. The transplant costs are all reimbursable by third party payers, or are covered by governmental funding in most countries. In 2011, the Organ Procurement and Transplantation Network (OPTN) reported that the number of pancreas transplants in general has been declining steadily since 2004. The decrease in transplant volume was directly related to reduced access to suitable organ donors and affected all pancreas transplant modalities [75, 23*0]. It is suspected but not causally proven that the advent of successful ITA has had major influence in reducing rates of PTA, as this group has traditionally had poorer graft functional outcome and increased perioperative mortality after PTA, and patients and endocrinologists have been drawn to ITA where all other efforts to stabilize poor glycemic control with intensive insulin have failed.

In the evolution of transplantation, islet transplantation may still be considered to be in its infancy, as the numbers of islet transplants carried out worldwide match activity achieved in whole pancreas transplantation in the mid1990s. According to the Collaborative Islet Transplant Registry 2010, 7th Annual Report, approximately 40 islet transplants were conducted in 1 year in North America, a fraction of the number of whole organ and pancreas transplants performed (Fig. 2a). Furthermore, as of December 31, 2010, the registry has collected data on 571 allogeneic islet transplant recipients from 1999-2009 [77]. The North American islet transplant centers accounted for $66 \%$ of recipients, whereas the JDRF European and Australian centers account for the remaining $34 \%$ [77]. Currently, in North America, only 22 transplant centers have active islet transplantation programs, and represent less than $10 \%$ of the total active transplant centers (Fig. 2b). Of the 47 islet transplant centers worldwide, only 11 perform more than 20 transplants a year, four of which are in North America and seven in Europe $[77,78]$. Islet transplantation continues to be limited to specialized centers as greater than half of the islet transplants performed were conducted in these 11 sites [78]. In North America, the Edmonton group continues to be the most active, with over 200 patients transplanted with over 400 intraportal procedures, and in 2012 alone 66 islet transplants were carried out (Shapiro, personal communication). This demonstrates the positive impact of governmental reimbursement, and the freedom of independence upon research funding alone to support a clinical transplant program.

Islet transplantation remains restricted to relatively few specialized global centers, and the procedure is associated with inherently substantial operating costs. As such, it is reasonable to debate cost to benefit ratio between islet and
Table 1 Worldwide islet transplant activity registered by CITR from $1999-2009$

CITR Seventh Annual Report (2011) [29, 71]. This data is kindly reproduced with express permission from the CITR registry

\begin{tabular}{lllllllll}
\hline & \multicolumn{2}{l}{ North America } & & & \multicolumn{2}{l}{ Europe/Australia } \\
\cline { 2 - 3 } & ITA & SIK/IAK & Total & & ITA & SIK/IAK & Total & Grand total \\
\hline Recipients & 341 & 35 & 376 & & 140 & 55 & 195 & 571 \\
Infusions & 650 & 66 & 716 & & 247 & 109 & 356 & 1,072 \\
Donors & 693 & 73 & 766 & & 295 & 126 & 421 & 1,187 \\
\hline
\end{tabular}


A

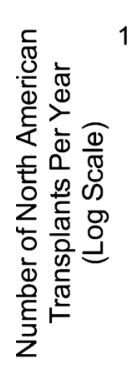

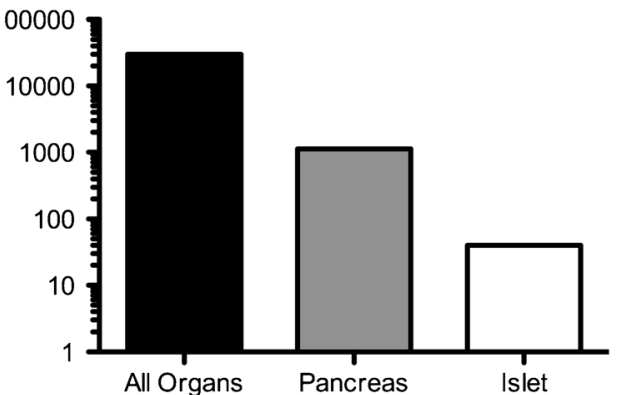

B

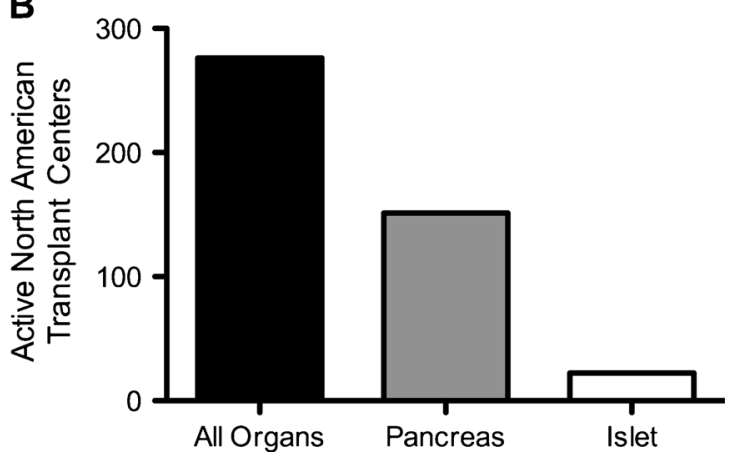

Fig. 2 Comparison of pancreas and islet transplants per year (a) and active centers in North America (b), based on the latest registry data (IPTR/OPTN/ UNOS/CITR). Log scale is used to display better the results $[77,75,23 \bullet \bullet$. This data is kindly reproduced with express permission from the IPTR and CITR registry

pancreas transplantation. Based on the 2011 UNOS report, the average billed cumulative cost in the United State for a whole organ pancreas transplant is approximately $\$ 270,000$, including donor organ procurement, transplant admission, physician transplant cost, and 180 days post-transplant admission, but these figures do not include immunosuppressive costs [75]. In contrast, islet transplantation is estimated to cost approximately $\$ 93,500$ - $\$ 120,000$; however, this figure does include the cost of 1 year of induction and maintenance immunosuppression, so is not directly comparable $[79,78]$. The cost breakdown for islet transplantation is more variable than pancreas transplant, as islet transplant costs also include initial hospitalization, screening and medical procedures $(\$ 28,500)$, donor organ procurement $(\$ 25,000-\$ 36,000)$ and islet manufacturing $(\$ 25,000-\$ 35,000)$ which usually require two to three pancreata as one in two islet isolations fail, and patients often require two islet infusions $(\$ 40,000)[79,78]$. It is estimated that $\$ 19,000$ is spent annually on patients receiving either a pancreas or islet transplantation encompassing costs associated with follow-up consultations, procedures and immunosuppressive drugs [78]. Thus, it would appear that the cumulative costs associated with islet transplant may be substantially lower than those associated with pancreas transplantation. The procedural cost breakdowns do not account for the substantially increased costs associated with complications, reoperative rates and prolonged intensive care unit and hospital stays associated with a sub-set of pancreas transplants. Pancreas transplantation is known to have significantly higher morbidity and mortality rates compared to islet transplantations, with complications including, thrombosis, bleeding, bile leakage, acute rejection and CMV infection [74, 23••, 78]. These medical interventions in addition to graft biopsies (which are not performed in islet transplantation) impose additional significant post-operative cost. In contrast, islet transplantation is associated with infrequent $(<10 \%)$ rates of portal vein thrombosis, exploratory laparotomy, and slightly more common bleeding and liver steatosis $[80,78]$.
A recent study by Beckwith et al provided a detailed health economic analysis of islet transplantation compared to exogenous insulin therapy. They concluded that the 20 -year cumulative cost of insulin treated patients and their secondary complications was $\$ 663,000$ with a quality-adjusted life years (QALYs) gain of 9.3, equating to an average costeffectiveness ratio of $\$ 71,000$ per QALY. In comparison to insulin therapy, islet transplantation had a 20-year cumulative cost $\$ 519,000$ with a QALYs gain of 10.9 , resulting in a cost-effective ratio of $\$ 47,000$ per QALY. When two islet infusions are required to achieve normoglycemia the costeffective ratio increases to $\$ 56,000$ per QALYs which is still well below that of standard insulin therapy and the acceptable $\$ 100,000$ threshold set by the United States [79]. These feasibility analyses demonstrate that islet transplantation can be cost-effective acutely post-transplant and indeed cost-saving in the long term.

In Europe and Canada islet transplantation is covered by their federal health care systems. However, in the United States, clinical islet transplantation remains experimental and investigational. The Clinical Islet Transplant (CIT) Consortium is conducting two pivotal Phase III clinical trials (CIT-06 \& CIT-07, Clinical Trials.gov NCT00468117 and NCT00434811, respectively) in selected specialized islet transplantation centers (Universities of Minnesota, Pennsylvania, Miami, Illinois at Chicago, California at San Francisco and Alberta, and Emory and Northwestern Universities), to support the FDA biological license application mandate. The anticipated results from these two trials will likely lead to successful licensure, allowing islet transplantation to be recognized as reimbursable therapy for patients with type 1 diabetes. The outcome of the biological licensing applications and in light of the significant advances that have been made in the new era of islet transplantation (2007 - 2010), which now demonstrated improved primary efficacy and safety outcomes with a 3-year insulin independent rate of $44 \%$, with fewer islet infusions and adverse events per patient $[77,30 \bullet \bullet, 62]$, provide further support that islet transplantation may be the more effective treatment 
modality for patients with diabetes who have already received a kidney transplant.

\section{PAK and IAK: Competition or Complementary Therapies?}

On the surface, it might seem that there is intense competition between whole pancreas and islet transplant programs, each competing for scarce pancreata, and each vying for pride of place, pitching robust glycemic control and insulin independence against relative morbidity of each approach. In practice, however, there is no great competition, and, indeed, in several of the leading centers, both whole pancreas and islet transplant programs coexist in harmony and are run by common surgical teams, and expertise in either modality can be of great benefit to the complementary team. Generally, there is not presently an acute shortage of pancreata, and the majority of pancreata are not procured for various reasons across the US. A prohibitive procurement fee of approximately $\$ 36,000$, lack of an on-site, geographically proximate surgically trained procurement team, and a potential need to ship marginal pancreata across great distances with consequent prolonged cold storage time all influence pancreas utilization rates presently. Younger pancreas donors of low body weight fair best in whole pancreas transplantation, whereas the obese younger donor with a more fatty pancreas may digest better for islet isolation, and be more prone to pancreatitis and peripancreatic infection if transplanted as a whole graft. Older donors often perform better in islet isolation rather than whole pancreas transplantation. Thus, organ sharing between whole pancreas and islet programs can provide both positive and rational assignment to improve overall organ utilization.

Improvement in recent outcomes in PAK and PTA through technical advances and more optimized immunosuppression mean that the comparative goalposts in pancreas and islet transplantation are constantly shifting [41, 81]. Ideally, SPK provides effective therapy for both renal insufficiency and T1DM at once in a single procedure. In reality, most deceased donors are not suitable for pancreas transplantation, and an increasing proportion of patients have access to living donation. PAK provides a unique opportunity to wait for a more ideal pancreas with increased likelihood of positive outcome. IAK may also be seen as a strategic procedure where an optimal viable islet mass is awaited before subjecting a patient to the implant procedure. The benefits of PAK versus IAK become less clear in selected specialized centers (Minnesota, Edmonton, Geneva, Lille and at UCSF) where insulinindependence at 5 years reaches $50 \%$ for ITA, which is very comparable to the current outcomes of PTA despite the donor selection bias towards PT $[32,55 \bullet]$. In our own center (Edmonton), we have relatively few suitable patients referred for PAK, but face an abundance of Canadian patients with unstable glycemic control that cannot be stabilized by intensive insulin, pumps or continuous glucose monitoring. It is these patients that are brought forward for ITA, and this will likely continue to be our dominant cellular transplant category.

Another factor influencing the number and modality of transplants performed is the program availability and associated cost. The previous section clearly explained the gap in number of accredited transplant centers for each modality and the corresponding cost for each procedure. These are major determinants in the type of treatment modality provided for patients and certainly skew the results.

\section{Conclusion}

The incident rate of T1DM among children under the age of 14 years is estimated to increase by $3 \%$ annually worldwide [82]. Despite continued efforts to optimize both monitoring and insulin treatment, a significant proportion of these individuals will progress to develop vascular complications and end-stage diabetic nephropathy.

SPK transplantation provides a logical and practical solution that resolves both renal failure and diabetes in a single combined operation, improving renal transplant half-life, reducing mortality, improving overall quality of life and significantly reducing healthcare costs $[83,84]$.

PAK transplantation is now a very attractive alternative for patients that have access to a suitable living donor kidney. In selected cases, pre-emptive kidney transplantation may be performed prior to initiation of hemodialysis [85].

PAK transplantation also decreases waiting time for patients and allows them to undergo surgery in an optimized clinical state. Still, occasional patients experience complications after renal transplant, which may preclude them from subsequent suitability for pancreas transplantation.

IAK transplantation is becoming a valid alternative to PAK, but is less tried and tested that ITA, and has not been strictly compared in a prospective randomized fashion to PAK. The perceived benefit is optimized (but imperfect) glycemic control that comes at a price of markedly decreased interventional morbidity.

PAK and IAK transplantation are seen as complementary and non-competing therapies, and both are valuable tools in the transplant armamentarium for treatment of complex patients. Both can provide insulin-independence, but PAK has more robust metabolic reserve, at least at early time-points, and IAK is achieved with low periprocedural morbidity. Focused large, multicenter studies will be required to compare effectively durable efficacy of the two approaches, but how such trials will be funded remains to be seen. It is certain that outcomes, immunosuppression and approaches for both IAK and PAK will continue to improve, as will interventional 
insulin and closed loop therapies, and progress in stem cell transplant and other alternative source therapies will further alter the balance over time, especially if these therapies can work in the absence of immunosuppression.

Acknowledgments Boris L. Gala-Lopez is supported through the Izaak Walton Killam Scholarship, and through a scholarship from the Alberta Diabetes Institute/University of Alberta. Andrew R. Pepper received a MITACS government of Canada-Industry-Academic partnership grant. A. M. James Shapiro is supported through Alberta Innovates Healthcare Solutions, and holds a Canada Research Chair in Transplantation Surgery and Regenerative Medicine funded through the Government of Canada. The clinical islet transplant program is supported through Alberta Healthcare, and receives research support through the Collaborative Islet Transplant Consortium (CIT) funded through the National Institutes of Health (NIH), National Institute of Digestive Diseases and Kidney (NIDDK) and through the National Institute of Allergy and Infectious Diseases (NIAID). The islet program also receives funding through the Juvenile Diabetes Research Foundation (JDRF), and through the Diabetes Research Institute Foundation of Canada (DRIFCan). All authors are also supported by AIHS CRIO Team Award \#201201154.

The authors thank the International Pancreas Transplant Registry for providing access to data published on their 2010 Annual report, as well as the Collaborative Islet Transplant Registry for granting access to their Seventh Annual Report.

The data reported in the 2011 Annual Data Report of the Organ Procurement and Transplantation Network and the US Scientific Registry of Transplant Recipients was kindly provided by the Minneapolis Medical Research Foundation and UNOS under contract with HHS/HRSA. The authors alone are responsible for reporting and interpreting these data, and the views expressed herein.

\section{Compliance with Ethics Guidelines}

Conflict of Interest Boris L. Gala-Lopez, Andrew R. Pepper, and A. M. James Shapiro declare that they have no conflict of interest.

Human and Animal Rights and Informed Consent This article does not contain any studies with human or animal subjects performed by any of the authors.

\section{References}

Papers of particular interest, published recently, have been highlighted as:

- Of importance

•- Of major importance

1. Marroquin CE, Edwards EB, Collins BH, Desai DM, TuttleNewhall JE, Kuo PC. Half-life analysis of pancreas and kidney transplants. Transplantation. 2005;80(2):272-5.

2. Waki K, Terasaki PI. Kidney graft and patient survival with and without a simultaneous pancreas utilizing contralateral kidneys from the same donor. Diabetes Care. 2006;29(7):1670-2. doi:10. 2337/dc05-2380.

3. Farney AC, Cho E, Schweitzer EJ, Dunkin B, Philosophe B, Colonna J, et al. Simultaneous cadaver pancreas living-donor kidney transplantation: a new approach for the type 1 diabetic uremic patient. Ann Surg. 2000;232(5):696-703.
4. Cavallari G, Neri F, Nardo B. Combined Kidney-Islet Transplantation. Understanding the Complexities of Kidney Transplantation. InTech; 2011.

5. Merani S, Shapiro AM. Current status of pancreatic islet transplantation. Clin Sci (Lond). 2006;110(6):611-25. doi:10.1042/ CS20050342.

6. Gerber PA, Pavlicek V, Demartines N, Zuellig R, Pfammatter T, Wuthrich R, et al. Simultaneous islet-kidney vs pancreas-kidney transplantation in type 1 diabetes mellitus: a 5 year single centre follow-up. Diabetologia. 2008;51(1):110-9. doi:10.1007/s00125007-0860-4. Excellent paper comparing outcomes of two relevant transplant modalities.

7. Giuseppe Cavallari FNaBN. Combined Kidney-Islet Transplantation. In: Ortiz PJ, editor. Understanding the Complexities of Kidney Transplantation. InTech; 2011.

8. Tan J, Yang S, Cai J, Guo J, Huang L, Wu Z, et al. Simultaneous islet and kidney transplantation in seven patients with type 1 diabetes and end-stage renal disease using a glucocorticoid-free immunosuppressive regimen with alemtuzumab induction. Diabetes. 2008;57(10):2666-71. doi:10.2337/db08-0358.

9. Young BY, Gill J, Huang E, Takemoto SK, Anastasi B, Shah T, et al. Living donor kidney versus simultaneous pancreas-kidney transplant in type I diabetics: an analysis of the OPTN/UNOS database. Clin J Am Soc Nephrol: CJASN. 2009;4(4):845-52. doi:10.2215/CJN.02250508. Excellent analysis paper on the benefits of living kidney donation.

10. Boggi U, Vistoli F, Del Chiaro M, Signori S, Coletti L, Morelli L, et al. Simultaneous cadaver pancreas-living donor kidney transplantation. Transplant Proc. 2004;36(3):577-9. doi:10.1016/j. transproceed.2004.03.072.

11.• Han DJ, Sutherland DE. Pancreas transplantation. Gut Liver. 2010;4(4):450-65. doi:10.5009/gnl.2010.4.4.450. Very complete review on the entire topic from the hand of pioneers. Multiple analysis are made on polemic issues around pancreas transplant, like sequential procedure, the main topic of this manuscript.

12. Squifflet JP, Gruessner RW, Sutherland DE. The history of pancreas transplantation: past, present and future. Acta Chir Belg. 2008;108(3):367-78.

13. Sutherland DE, Gruessner RW, Gruessner AC. Pancreas transplantation for treatment of diabetes mellitus. World J Surg. 2001;25(4): 487-96. doi:10.1007/s002680020342.

14. Bazerbachi F, Selzner M, Boehnert MU, Marquez MA, Norgate A, McGilvray ID, et al. Thymoglobulin versus basiliximab induction therapy for simultaneous kidney-pancreas transplantation: impact on rejection, graft function, and long-term outcome. Transplantation. 2011;92(9):1039-43. doi:10.1097/TP.0b013e3182313e4f.

15. Muthusamy AS, Vaidya AC, Sinha S, Roy D, Elker DE, Friend PJ. Alemtuzumab induction and steroid-free maintenance immunosuppression in pancreas transplantation. Am J transplant: Off J Am Soc Transplant Am Soc Transplant Surg. 2008;8(10):2126-31. doi:10. 1111/j.1600-6143.2008.02373.x.

16. Stegall MD, Kim DY, Prieto M, Cohen AJ, Griffin MD, Schwab TR, et al. Thymoglobulin induction decreases rejection in solitary pancreas transplantation. Transplantation. 2001;72(10): 1671-5

17. Philosophe B, Farney AC, Schweitzer EJ, Colonna JO, Jarrell BE, Krishnamurthi V, et al. Superiority of portal venous drainage over systemic venous drainage in pancreas transplantation: a retrospective study. Ann Surg. 2001;234(5):689-96.

18. Bazerbachi F, Selzner M, Marquez MA, Norgate A, Aslani N, McGilvray ID, et al. Portal venous versus systemic venous drainage of pancreas grafts: impact on long-term results. Am J Transplant: Off J Am Soc Transplant Am Soc Transplant Surg. 2012;12(1): 226-32. doi:10.1111/j.1600-6143.2011.03756.x.

19. Sutherland DE, Gruessner A, Hering BJ. Beta-cell replacement therapy (pancreas and islet transplantation) for treatment of diabetes 
mellitus: an integrated approach. Endocrinol Metabol Clin N Am. 2004;33(1):135-48, x. doi:10.1016/S0889-8529(03)00099-9.

20. Fridell JA, Mangus RS, Hollinger EF, Taber TE, Goble ML, Mohler $\mathrm{E}$, et al. The case for pancreas after kidney transplantation. Clin Transplant. 2009;23(4):447-53. doi:10.1111/j.1399-0012.2009. 00996.x. Very complete clinical paper discussing the benefits and the new role of pancreas after kidney transplant.

21. Hariharan S, Pirsch JD, Lu CY, Chan L, Pesavento TE, Alexander $\mathrm{S}$, et al. Pancreas after kidney transplantation. J Am Soc Nephrol: JASN. 2002;13(4):1109-18.

22. Sutherland DE. Current status of beta-cell replacement therapy (pancreas and islet transplantation) for treatment of diabetes mellitus. Transplant Proc. 2003;35(5):1625-7.

23.• Gruessner AC, Gruessner RW. Pancreas transplant outcomes for United States and non United States cases as reported to the United Network for Organ Sharing and the International Pancreas Transplant Registry as of. Clin Transpl. 2011;2012:23-40. IPTR 2011 Annual report with some of the most updated clinical information in the US.

24. Gruessner AC, Sutherland DE. Pancreas transplant outcomes for United States (US) and non-US cases as reported to the United Network for Organ Sharing (UNOS) and the International Pancreas Transplant Registry (IPTR) as of June 2004. Clin Transplant. 2005;19(4):433-55. doi:10.1111/j.1399-0012.2005. 00378.x.

25. Muthusamy AS, Mumford L, Hudson A, Fuggle SV, Friend PJ. Pancreas transplantation from donors after circulatory death from the United Kingdom. Am J Transplant: Off J Am Soc Transplant Am Soc Transplant Surg. 2013;13(3): 824. doi:10.1111/ajt.12037.

26. Shapiro AM, Lakey JR, Ryan EA, Korbutt GS, Toth E, Warnock $\mathrm{GL}$, et al. Islet transplantation in seven patients with type 1 diabetes mellitus using a glucocorticoid-free immunosuppressive regimen. New Engl J Med. 2000;343(4):230-8. doi:10. 1056/NEJM200007273430401.

27. Ryan EA, Lakey JR, Paty BW, Imes S, Korbutt GS, Kneteman NM, et al. Successful islet transplantation: continued insulin reserve provides long-term glycemic control. Diabetes. 2002;51(7):2148-57.

28. Ryan EA, Paty BW, Senior PA, Bigam D, Alfadhli E, Kneteman NM, et al. Five-year follow-up after clinical islet transplantation. Diabetes. 2005;54(7):2060-9.

29. Shapiro AM, Ricordi C, Hering BJ, Auchincloss H, Lindblad R, Robertson RP, et al. International trial of the Edmonton protocol for islet transplantation. New Engl J Med. 2006;355(13):1318-30. doi: 10.1056/NEJMoa061267.

30.• Barton FB, Rickels MR, Alejandro R, Hering BJ, Wease S, Naziruddin B, et al. Improvement in outcomes of clinical islet transplantation: 1999-2010. Diabetes Care. 2012;35(7):1436-45. doi:10.2337/dc12-0063. CITR 2011 Annual report with the most updated clinical information on islet transplantation.

31. Bellin MD, Barton FB, Heitman A, Harmon JV, Kandaswamy R, Balamurugan $\mathrm{AN}$, et al. Potent induction immunotherapy promotes long-term insulin independence after islet transplantation in type 1 diabetes. Am J Transplant: Off J Am Soc Transplant Am Soc Transplant Surg. 2012;12(6):1576-83. doi:10.1111/j.1600-6143. 2011.03977.x. Excellent paper showing the long-term benefits of T-cell depletion in islet transplantation.

32. Shapiro AM. Strategies toward single-donor islets of Langerhans transplantation. Curr Opin Organ Transplant. 2011;16(6):627-31. doi:10.1097/MOT.0b013e32834cfb84.

33. Berney T, Bucher P, Mathe Z, Andres A, Bosco D, Mage R, et al. Islet of Langerhans allogeneic transplantation at the University of Geneva in the steroid free era in islet after kidney and simultaneous islet-kidney transplantations. Transplant Proc. 2004;36(4):1121-2. doi:10.1016/j.transproceed.2004.04.027.
34. Borot S, Niclauss N, Wojtusciszyn A, Brault C, DemuylderMischler S, Muller Y, et al. Impact of the number of infusions on 2 -year results of islet-after-kidney transplantation in the GRAGIL network. Transplantation. 2011;92(9):1031-8. doi:10.1097/TP. 0b013e318230c236.

35. Friberg AS, Lundgren T, Malm H, Felldin M, Nilsson B, Jenssen T, et al. Transplanted functional islet mass: donor, islet preparation, and recipient factors influence early graft function in islet-afterkidney patients. Transplantation. 2012;93(6):632-8. doi:10.1097/ TP.0b013e3182455912.

36. Feng S, Goodrich NP, Bragg-Gresham JL, Dykstra DM, Punch JD, DebRoy MA, et al. Characteristics associated with liver graft failure: the concept of a donor risk index. Am J Transplant: Off J Am Soc Transplant Am Soc Transplant Surg. 2006;6(4):783-90. doi: 10.1111/j.1600-6143.2006.01242.x.

37. Rao PS, Schaubel DE, Guidinger MK, Andreoni KA, Wolfe RA, Merion RM, et al. A comprehensive risk quantification score for deceased donor kidneys: the kidney donor risk index. Transplantation. 2009;88(2):231-6. doi:10.1097/TP. 0b013e3181ac620b.

38. Axelrod DA, Sung RS, Meyer KH, Wolfe RA, Kaufman DB. Systematic evaluation of pancreas allograft quality, outcomes and geographic variation in utilization. Am J Transplant: Off J Am Soc Transplant Am Soc Transplant Surg. 2010;10(4):837-45. doi:10. 1111/j.1600-6143.2009.02996.x. First analysis on the potential benefits of DRI for pancreas transplantation.

39. Paty BW, Koh A, Senior P. Pancreas and islet transplantation. Can J Diabetes. 2013;37:S94-6. doi:10.1016/j.jcjd.2013.01.028.

40. Gruessner AC, Sutherland DE. Access to pancreas transplantation should not be restricted because of age: invited commentary on Schenker et al. Transplant Int: Off J Eur Soc Organ Transplant. 2011;24(2):134-5. doi:10.1111/j.1432-2277.2010.01180.x.

41. Kleinclauss F, Fauda M, Sutherland DE, Kleinclauss C, Gruessner RW, Matas AJ, et al. Pancreas after living donor kidney transplants in diabetic patients: impact on long-term kidney graft function. Clin Transplant. 2009;23(4):437-46. doi:10.1111/j.1399-0012.2009. 00998.x.

42.• Gruessner AC, Sutherland DE, Gruessner RW. Pancreas transplantation in the United States: a review. Curr Opin Organ Transplant. 2010;15(1):93-101. doi:10.1097/MOT.0b013e32833552d2. Excellent update on pancreas transplant activity.

43. Halloran PF. Immunosuppressive drugs for kidney transplantation. New Engl J Med. 2004;351(26):2715-29. doi:10.1056/ NEJMra033540.

44. Gruessner RW, Kandaswamy R, Humar A, Gruessner AC, Sutherland DE. Calcineurin inhibitor- and steroid-free immunosuppression in pancreas-kidney and solitary pancreas transplantation. Transplantation. 2005;79(9):1184-9.

45. Jang HJ, Kim SC, Han DJ. Comparison of pancreas transplantation outcome between the cyclosporine and tacrolimus eras. Transplant Proc. 2000;32(7):2470-1.

46. Zagozda M, Sarnecka A, Durlik M. Microchimerism after pancreas and kidney transplantation-a review. Ann Transplant: Q Polish Transplant Soc. 2011;16(4):134-7.

47. Burke 3rd GW, Vendrame F, Pileggi A, Ciancio G, Reijonen H, Pugliese A. Recurrence of autoimmunity following pancreas transplantation. Curr Diabetes Rep. 2011;11(5):413-9. doi:10.1007/ s11892-011-0206-y.

48. Diamantopoulos S, Allende G, Ferreira JM, Ciancio G, Burke GW, Pugliese A. Retrospective assessment of islet cell autoantibodies in pancreas organ donors. Diabetes Care. 2008;31(9):1741-2. doi:10. 2337/dc08-0652.

49. Laughlin E, Burke G, Pugliese A, Falk B, Nepom G. Recurrence of autoreactive antigen-specific CD4+ T cells in autoimmune diabetes after pancreas transplantation. Clin Immunol. 2008;128(1):23-30. doi:10.1016/j.clim.2008.03.459. 
50. Pugliese A, Reijonen HK, Nepom J, Burke 3rd GW. Recurrence of autoimmunity in pancreas transplant patients: research update. Diabetes Manag. 2011;1(2):229-38. doi:10. 2217/dmt.10.21.

51. Gala-Lopez B, Pepper AR, Shapiro AM. Biol Agents Islet Transplant. Curr Diabetes Rep. 2013. doi:10.1007/s11892-0130414-8.

52.• Kandaswamy R, Stock PG, Skeans MA, Gustafson SK, Sleeman EF, Wainright JL, et al. OPTN/SRTR 2011 annual data report: pancreas. Am J Transplant: Off J Am Soc Transplant Am Soc Transplant Surg. 2013;13 Suppl 1:47-72. doi:10.1111/ajt.12020. OPTN/SRTR 2011 annual report with some of the most updated clinical information in the US.

53. Plesner A, Verchere CB. Advances and challenges in islet transplantation: islet procurement rates and lessons learned from suboptimal islet transplantation. J Transplant. 2011;2011:979527. doi:10. 1155/2011/979527.

54. Shapiro AM. A historical perspective on experimental and clinical islet transplantation. In: Shapiro AM, Shaw JA, editors. Islet transplantation and beta cell replacement therapy. New York: Informa Healthcare; 2007.

55. Shapiro AM. State of the art of clinical islet transplantation and novel protocols of immunosuppression. Curr Diabetes Rep. 2011;11(5):345-54. doi:10.1007/s11892-011-0217-8. Excellent review on the new protocols used to improve outcomes of islet transplantation.

56. Naziruddin B, Wease S, Stablein D, Barton FB, Berney T, Rickels $\mathrm{MR}$, et al. HLA class I sensitization in islet transplant recipients: report from the Collaborative Islet Transplant Registry. Cell Transplant. 2012;21(5):901-8.

57. Gala-Lopez BL, Senior PA, Koh A, Kashkoush SM, Kawahara T, Kin T, et al. Late cytomegalovirus transmission and impact of T-depletion in clinical islet transplantation. Am J Transplant: Off J Am Soc Transplant Am Soc Transplant Surg. 2011;11(12):2708-14. doi:10.1111/j.16006143.2011.03724.x.

58. Gala-Lopez B, Kin T, O'Gorman D, Pepper AR, Senior P, Humar A, et al. Microbial contamination of clinical islet transplant preparations is associated with very low risk of infection. Diabetes Technol Ther. 2013;15(4):323-7.

59. Koh A, Senior P, Salam A, Kin T, Imes S, Dinyari P, et al. Insulin-heparin infusions peritransplant substantially improve single-donor clinical islet transplant success. Transplantation. 2010;89(4):465-71.

60. Pepper AR, Gala-Lopez B, Ziff O, Shapiro AM. Revascularization of transplanted pancreatic islets and role of the transplantation site. Clin Dev Immunol. 2013;2013:352315. doi:10.1155/ 2013/352315.

61. Senior PA, Kin T, Shapiro AMJ, Koh A. Islet transplantation at the University of Alberta: status update and review of progress over the last decade. Can J Diabetes. 2012;36:32-7. One of the latest updates of transplant activity, associated morbidity and long-term results from the Edmonton group.

62. Pepper AR, Gala-Lopez B, Ziff O, Shapiro AJ. Current status of clinical islet transplantation. World J Transplant. 2013;3(4):48-53. doi:10.5500/wjt.v3.i4.48.

63. Senior PAKT, Shapiro AMJ, Koh A. Islet transplantation at the University of Alberta: status update and review of progress over the last decade. Can J Diabetes. 2012;36:32-7. doi:10.1016/j.jcjd.2012. 01.002 .

64. Andres A, Toso C, Morel P, Demuylder-Mischler S, Bosco D, Baertschiger R, et al. Impairment of renal function after islet transplant alone or islet-after-kidney transplantation using a sirolimus/ tacrolimus-based immunosuppressive regimen. Transplant Int: Off J Eur Soc Organ Transplant. 2005;18(11):1226-30. doi:10.1111/j. 1432-2277.2005.00194.x.
65. Maffi P, Bertuzzi F, De Taddeo F, Magistretti P, Nano R, Fiorina P, et al. Kidney function after islet transplant alone in type 1 diabetes: impact of immunosuppressive therapy on progression of diabetic nephropathy. Diabetes Care. 2007;30(5):1150 5. doi:10.2337/dc06-1794.

66. Campbell PM, Senior PA, Salam A, Labranche K, Bigam DL, Kneteman NM, et al. High risk of sensitization after failed islet transplantation. Am J Transplant: Off J Am Soc Transplant Am Soc Transplant Surg. 2007;7(10):2311-7. doi:10.1111/j.1600-6143. 2007.01923.x.

67. Ferrari-Lacraz S, Berney T, Morel P, Marangon N, Hadaya $\mathrm{K}$, Demuylder-Mischler S, et al. Low risk of anti-human leukocyte antigen antibody sensitization after combined kidney and islet transplantation. Transplantation. 2008;86(2): $357-9$.

68. Froud T, Yrizarry JM, Alejandro R, Ricordi C. Use of D-STAT to prevent bleeding following percutaneous transhepatic intraportal islet transplantation. Cell Transplant. 2004;13(1): 55-9.

69. Kawahara T, Kin T, Kashkoush S, Gala-Lopez B, Bigam DL, Kneteman NM, et al. Portal vein thrombosis is a potentially preventable complication in clinical islet transplantation. Am J Transplant: Off J Am Soc Transplant Am Soc Transplant Surg. 2011;11(12):2700-7. doi:10.1111/j.1600-6143.2011.03717.x.

70. Owen RJ, Ryan EA, O'Kelly K, Lakey JR, McCarthy MC, Paty BW, et al. Percutaneous transhepatic pancreatic islet cell transplantation in type 1 diabetes mellitus: radiologic aspects. Radiology. 2003;229(1):165-70. doi:10.1148/radiol. 2291021632.

71. Villiger P, Ryan EA, Owen R, O'Kelly K, Oberholzer J, Al Saif F, et al. Prevention of bleeding after islet transplantation: lessons learned from a multivariate analysis of 132 cases at a single institution. Am J Transplant: Off J Am Soc Transplant Am Soc Transplant Surg. 2005;5(12):2992-8. doi:10.1111/j.1600-6143. 2005.01108.x.

72. Walsh RM, Saavedra JR, Lentz G, Guerron AD, Scheman J, Stevens $T$, et al. Improved quality of life following total pancreatectomy and auto-islet transplantation for chronic pancreatitis. J gastrointest Surg: Off J Soc Surg Aliment Tract. 2012;16(8): 1469-77. doi:10.1007/s11605-012-1914-6.

73. Deng S, Markmann JF, Rickels M, Yeh H, Kim JI, Lian MM, et al. Islet alone versus islet after kidney transplantation: metabolic outcomes and islet graft survival. Transplantation. 2009;88(6):820-5. doi:10.1097/TP.0b013e3181b4bdbb.

74. Boggi U, Vistoli F, Egidi FM, Marchetti P, De Lio N, Perrone V, et al. Transplantation of the pancreas. Curr Diabetes Rep. 2012;12(5):568-79. doi:10.1007/s11892-012-0293-4.

75. (SRTR) OPaTNOaSRoTR. OPTN/SRTR 2011 Annual Data Report. In: Health and Human Services HRaSA, Healthcare Systems Bureau, Divison of Transplantation, editor. Rockville, MD2012.

76. Report CORRA. Treatment of End-Stage Organ Failure in Canada, 2001 to 2010. In: Information CIfH, editor. Ottawa, ON2011.

77. Seventh Annual Data Report [database on the Internet] 2011. Available from: http://www.citregistry.org/reports/reports.htm. Accessed:

78. Vrochides D, Paraskevas S, Papanikolaou V. Transplantation for type 1 diabetes mellitus. Whole organ or islets? Hippokratia. 2009;13(1):6-8.

79. Beckwith J, Nyman JA, Flanagan B, Schrover R, Schuurman HJ. A health economic analysis of clinical islet transplantation. Clin Transplant. 2012;26(1):23-33. doi:10.1111/j.1399-0012.2011. 01411.x.

80. Vardanyan M, Parkin E, Gruessner C, Rodriguez Rilo HL. Pancreas vs. islet transplantation: a call on the future. Curr 
Opin Organ transplant. 2010;15(1):124-30. doi:10.1097/ MOT.0b013e32833553f8.

81. Robertson RP, Davis C, Larsen J, Stratta R, Sutherland DE. Pancreas and islet transplantation in type 1 diabetes. Diabetes Care. 2006;29(4):935.

82. Federation ID. IDF diabetes atlas. Brussels: International Diabetes Federation; 2013.

83. Knoll GA, Nichol G. Dialysis, kidney transplantation, or pancreas transplantation for patients with diabetes mellitus and renal failure: a decision analysis of treatment options. J Am Soc Nephrol: JASN. 2003;14(2):500-15.

84. Lenisa L, Castoldi R, Socci C, Motta F, Ferrari G, Spotti D, et al. Cost-effective treatment for diabetic end-stage renal disease: dialysis, kidney, or kidney-pancreas transplantation? Transplant Proc. 1995;27(6):3108-13.

85. Davis CL. Preemptive transplantation and the transplant first initiative. Curr Opin Nephrol Hypertens. 2010;19(6):592-7. doi:10. 1097/MNH.0b013e32833e04f5. 